\title{
Symmetric Fundamental Expansions to Schur Positivity
}

\author{
Austin Roberts ${ }^{1}$ \\ ${ }^{1}$ Highline College, Des Moines, WA / 1QBit Information Technologies, Vancouver, BC
}

\begin{abstract}
We consider families of quasisymmetric functions with the property that if a symmetric function $f$ is a positive sum of functions in one of these families, then $f$ is necessarily a positive sum of Schur functions. Furthermore, in each of the families studied, we give a combinatorial description of the Schur coefficients of $f$. We organize six such families into a poset, where functions in higher families in the poset are always positive integer sums of functions in each of the lower families.

Résumé. Nous considérons les familles de fonctions quasi-symétriques avec la propriété suivante: si une fonction symétrique $f$ est une somme positive de fonctions dans une de ces familles, alors $f$ est nécessairement une somme positive de fonctions de Schur. En outre, dans chacune des familles étudiées, nous donnons une description combinatoire des coefficients de Schur de $f$. Nous organisons six de ces familles dans un ensemble ordonné, où les fonctions des familles plus élevées dans le poset sont toujours des sommes d'entiers positifs de fonctions dans chacune des familles inférieurs.
\end{abstract}

Keywords. Schur functions, symmetric functions, quasisymmetric, positivity, RSK, Knuth equivalence

\section{Introduction}

In this extended abstract, we consider families of quasisymmetric functions with the property that if a symmetric function $f$ is a positive sum of functions in one of these families, then $f$ is necessarily a positive sum of Schur functions. Furthermore, in each of the families studied, we give a combinatorial description of the Schur coefficients of $f$. We organize six such families into a poset, where functions in higher families in the poset are positive integer sums of functions in each of the lower families. This poset includes the Schur functions, quasisymmetric Schur functions, the fundamental quasisymmetric generating functions of shifted dual equivalence classes $\left\{f^{(h)}\right\}$, as well as three new families of functions $\left\{f^{(k)}\right\}$ for $k=0,1,2$ conjecturing that $\left\{f^{(2)}\right\}$ forms a basis for the vector space of quasisymmetric functions.

The poset of functions is first realized using a poset of equivalence relations. Each of the families is defined as sums of fundamental quasisymmetric functions over equivalence classes on standard Young tableaux of fixed partition shape $\lambda$, $\operatorname{SYT}(\lambda)$. A higher position in our poset represents a coarser relation. We then use the Robinson-Schensted-Knuth (RSK) correspondence to create equivalence classes on permutations. An illustration of said poset can be found in Figure 1 .

While detailed definitions will be given later, we can describe the main results as follows. In Gessel. 1984), Gessel used the set $\operatorname{SYT}(\lambda)$ to express the Schur function $s_{\lambda}$ as, 

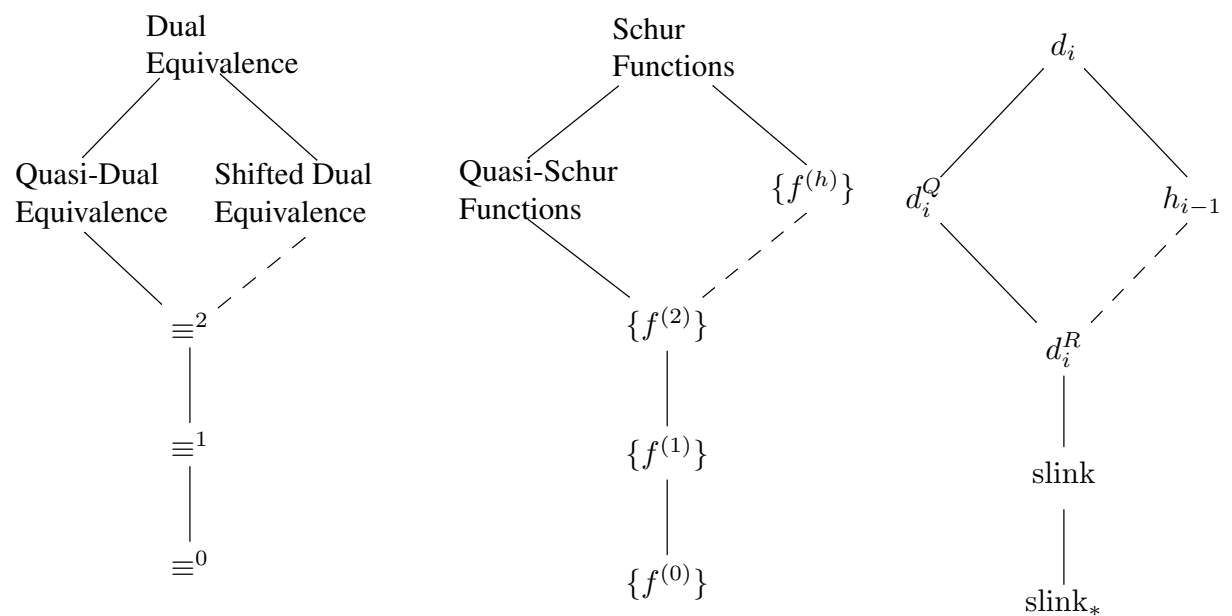

Fig. 1: At left, the poset of equivalence classes. At center, the associated quasisymmetric functions of the equivalence class. At right, the generators of the equivalence relations. The dashed lines denote that a simple transformation is required.

$$
s_{\lambda}=\sum_{T \in \operatorname{SYT}(\lambda)} F_{\mathrm{ID}(T)}(X)
$$

where $F_{\mathrm{ID}(T)}(X)$ is the fundamental quasisymmetric function

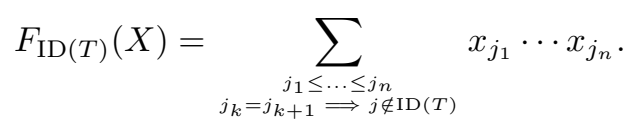

Here, $\operatorname{ID}(T)$ is the inverse descent set of $T$. We define three equivalence relations $\equiv^{0}, \equiv^{1}$, and $\equiv^{2}$ on $\operatorname{SYT}(\lambda)$, each a refinement of the next. One particularly nice equivalence class of $\equiv^{0}$ and $\equiv^{1}$ is the class of a single element, the superstandard tableau $U_{\lambda}$. Here, $\lambda$ is a partition of $n$ and $U_{\lambda} \in \operatorname{SYT}(n)$ is the standard Young tableau formed by placing the numbers 1 through $n$, in order, in the lowest row possible. The main result can now be stated as follows.

Theorem 1.1 If $k=0,1$, or 2 , and $\mathcal{C} \subset \operatorname{SYT}(n)$ is a disjoint union of equivalence classes of ${ }^{k}$ such that $f=\sum_{T \in \mathcal{C}} F_{\mathrm{ID}(T)}$ is a symmetric function, then

$$
f=\sum_{\lambda \vdash n} c_{\lambda} s_{\lambda}
$$

where $c_{\lambda}$ is the multiplicity of $U_{\lambda}$ in $\mathcal{C}$.

Corollary 2.11 then generalizes this theorem to permutations. We conjecture that the family of fundamental quasisymmetric generating functions over $\equiv^{2},\left\{f^{(2)}\right\}$, form a basis for the quasisymmetric functions. 
We then connect our work to two well-studied families of functions. First is the set of quasisymmetric Schur functions, which — as the name suggests - are a quasisymmetric analogue of the Schur functions. Quasisymmetric Schur functions can be realized as a sum over standard reverse composition tableaux of a fixed composition shape, $\operatorname{SRCT}(\alpha)$. In Proposition 3.2 we describe a transitive action on $\operatorname{SRCT}(\alpha)$. We show in Proposition 3.3 that Mason's bijection sends our transitive action on $\operatorname{SRCT}(\alpha)$ to a subset of dual Knuth equivalences, which we term quasi-dual equivalences. In Lemma 3.5. we show that quasi-dual equivalence is a coarsening of $\equiv^{k}$, for each $k=0,1,2$. Hence the quasisymmetric Schur functions are positive integer sums of functions in $\left\{f^{(k)}\right\}$.

Finally, we consider shifted dual equivalence. Similar to the quasisymmetric Schur functions, Proposition 4.4 states that the generators for shifted dual equivalence strictly contain the generators for $\equiv^{2}$ after applying a simple involution. In Proposition 4.8, we further show that the set of row reading words of shifted standard Young tableaux with a fixed shape comprise an equivalence class of $\equiv^{2}$.

The paper is organized as follows. After preliminary lemmas and definitions, Section 2 defines the equivalence relations $\equiv^{k}$ and proves Theorem 1.1 . Section 2.3 generalizes results to permutations, with the main result generalized in Corollary 2.3 Section 3 is dedicated to the previously mentioned results related to quasisymmetric Schur functions. Finally, Section 4, which is independent of Section 3 , is dedicated to proving the results related to shifted dual equivalence.

\section{The equivalence relations}

\subsection{Preliminaries}

A composition $\alpha=\left(\alpha_{1}, \alpha_{2}, \ldots, \alpha_{k}\right)$ is a finite sequence of positive integers. We write $\alpha \vDash n$ and say that $\alpha$ is a composition of $n$ if $\sum_{i} \alpha_{i}=n$. A partition $\lambda=\left(\lambda_{1}, \lambda_{2}, \ldots, \lambda_{k}\right)$ is a weakly decreasing composition. We write $\lambda \vdash n$ and say $\lambda$ is a partition of $n$ if $\sum_{i} \lambda_{i}=n$. In this extended abstract, $\lambda$ will always be a partition of $n, \alpha$ will always be a composition of $n$, and $\lambda(\alpha)$ is the composition achieved by sorting the parts of $\alpha$ into weakly decreasing order.

The diagram of a composition or a partition will always be given in French notation. That is, the diagram is given by a set of left justified cells, drawn as boxes, in the Cartesian plane, where the $i^{\text {th }}$ row from bottom to top has $\alpha_{i}$ or $\lambda_{i}$ cells, respectively. The bottom left cell can be assumed to be the origin. The underlying composition or partition is then referred to as the shape of the diagram. Given a partition $\lambda$, the conjugate of $\lambda$, denoted $\lambda^{\prime}$, is the partition whose $i^{\text {th }}$ column has $\lambda_{i}$ many cells.

A filling $T$ of a diagram is a function that assigns a positive integer to each box of the diagram. A filling is standard if it uses every number in $[n]=\{1, \ldots, n\}$ exactly once. The set of standard Young tableaux of shape $\lambda$, denoted $\operatorname{SYT}(\lambda)$, is the set of standard fillings of $\lambda$ that are increasing up columns and across rows from left to right. The union of $\operatorname{SYT}(\lambda)$ over all $\lambda \vdash n$ is denoted $\operatorname{SYT}(n)$. The superstandard tableau of shape $\lambda$, denoted $U_{\lambda}$, is the standard Young tableau formed by placing the numbers 1 through $n$, in order, in the lowest row possible. The row reading word of a filling, $\operatorname{rw}(T)$, is obtained by reading the values of each row from left to right, starting with the top row and continuing down. The row reading word will always be used for standard Young tableaux, as well as for ordering their cells, but other reading words will be introduced for other types of fillings as needed.

In this abstract, permutations are always presented in one-line notation. Given a permutation $\pi \in S_{n}$, the inverse descent set of $\pi, \operatorname{ID}(\pi)$, is the set of $i \in[n-1]$ such that $i$ occurs after $i+1$ in $\pi$. Alternately, we can encode $\operatorname{ID}(\pi)$ with the composition $\beta(\pi)=\left(\beta_{1}(\pi), \ldots, \beta_{k}(\pi)\right) \vDash n$, letting $\beta_{i}$ be the difference 
between the $i$ and $(i-1)^{t h}$ numbers in $\operatorname{ID}(\pi) \cup\{n\}$, where the zero ${ }^{t h}$ number is always treated as 0 . As further notation, $\beta_{i, j}=\left(\beta_{i}, \beta_{j}\right)$. Given a standard filling $T$, define $\operatorname{ID}(T)$ and $\beta(T)$ via the reading word of $T$. Define the $i^{\text {th }}$ run of $T,\left.T\right|_{(a, b]}$, to be the restriction of $T$ to the set of cells with values in the integer interval $(a, b]$, where $a$ and $b$ are the $i-1$ and $i^{t h}$ numbers in $\operatorname{ID}(T) \cup\{n\}$, respectively. Notice that $b-a=\beta_{i}$. Similarly, the first $j$ runs of $T$ is the standard Young tableau achieved by taking the union of the first $j$ runs of $T$. Notice that for any $T \in \operatorname{SYT}(\lambda)$, the cells of the runs of $T$ fully determine $T$. For this reason, it can be helpful to consider the unstandard Young tableau, UYT, achieved by placing $i$ 's in each cell of the $i^{\text {th }}$ run. See Figure 2 for examples.

\begin{tabular}{|c|c|c|c|}
\hline 9 & 3 & 6 & 3 \\
\hline \begin{tabular}{|l|l|l|l|}
5 & 6 & 7 & 8 \\
\end{tabular} & \begin{tabular}{|l|l|l|l|}
2 & 2 & 2 & 2 \\
\end{tabular} & \begin{tabular}{|l|l|l|l|}
3 & 4 & 8 & 9 \\
\end{tabular} & \begin{tabular}{|l|l|l|l|}
2 & 2 & 4 & 5 \\
\end{tabular} \\
\hline \begin{tabular}{|l|l|l|l|}
1 & 2 & 3 & 4 \\
\end{tabular} & \begin{tabular}{|l|l|l|l|}
1 & 1 & 1 & 1 \\
\end{tabular} & \begin{tabular}{|l|l|l|l|}
1 & 2 & 5 & 7 \\
\end{tabular} & \begin{tabular}{|l|l|l|l|}
1 & 1 & 2 & 3 \\
\end{tabular} \\
\hline
\end{tabular}

Fig. 2: Two tableaux in $\operatorname{SYT}(\lambda)$ with their associated fillings in $\operatorname{UYT}(\lambda)$. Here, $\lambda=(4,4,1)$. At left, $U_{\lambda}$ with $\operatorname{rw}\left(U_{\lambda}\right)=956781234, \operatorname{ID}\left(U_{\lambda}\right)=\{4,8\}$, and $\beta(T)=(4,4,1)$. At right, tableau $T$ with $\operatorname{rw}(T)=634891257$, $\operatorname{ID}(T)=\{2,5,7\}$ and $\beta(T)=(2,3,2,2)$.

The RSK correspondence provides a bijection that sends each $\pi \in S_{n}$ to an ordered pair of standard Young tableaux $(P(\pi), Q(\pi))$, where $P(\pi)$ and $Q(\pi)$ are both in $\operatorname{SYT}(\lambda)$ for some $\lambda \vdash n$. Here, $P(\pi)$ is termed the insertion tableau and $Q(\pi)$ is termed the recording tableau. Two permutations with the same $P$ tableau are Knuth equivalent, while two permutations with the same $Q$ tableau are dual Knuth equivalent or just dual equivalent. We will assume familiarity with the RSK correspondence.

Let $\phi$ be a given function on standard Young tableaux that restricts to an involution on each $\operatorname{SYT}(\lambda)$. Define the action of $\phi$ on $S_{n}$ via the insertion tableaux to be the function that sends $\pi \in S_{n}$ to the unique $\pi^{\prime}$ such that $P\left(\pi^{\prime}\right)=\phi \circ P(\pi)$ and $Q\left(\pi^{\prime}\right)=Q(\pi)$. In the context of permutations, the role of $U_{\lambda}$ will be replaced by the set of standardized Yamanouchi words of shape $\lambda$, defined as

$$
\operatorname{SYam}(\lambda)=\left\{\pi \in S_{n}: P(\pi)=U_{\lambda}\right\}
$$

As an aside, we may also algorithmically generate $\operatorname{SYam}(\lambda)$. First generate the set of words with $\lambda_{i}$ many $i$ 's such that when reading in reverse order there are always weakly more $i$ 's than $i+1$ 's. These are the Yamanouchi words of weight $\lambda$. To achieve $\operatorname{SYam}(\lambda)$, turn each word into a permutation with the same relative order, where an $i$ that occurs earlier in reading order is considered smaller than an $i$ with a larger index. For example, the Yamanouchi words 111, 121, 211, and 321 correspond to the standardized Yamanouchi words 123, 132, 312, and 321, respectively.

We may generate dual equivalence classes of permutations as follows. Given a permutation in $S_{n}$ expressed in one-line notation, define an elementary dual equivalence as an involution $d_{i}$ that interchanges the values $i-1, i$, and $i+1$ as

$$
\begin{aligned}
d_{i}(\ldots i \ldots i-1 \ldots i+1 \ldots) & =(\ldots i+1 \ldots i-1 \ldots i \ldots), \\
d_{i}(\ldots i \ldots i+1 \ldots i-1 \ldots) & =(\ldots i-1 \ldots i+1 \ldots i \ldots),
\end{aligned}
$$

and as the identity when $i$ is positioned between $i-1$ and $i+1$. Two words are then dual equivalent if one may be transformed into the other by successive elementary dual equivalences. As an example, 21345 is dual equivalent to 51234 because $d_{4}\left(d_{3}\left(d_{2}(21345)\right)\right)=d_{4}\left(d_{3}(31245)\right)=d_{4}(41235)=51234$.

For a permutation $\pi \in S_{n}$, let $\left.\pi\right|_{I}$ be the subword consisting of values in the interval $I$, while $\pi_{I}$ is the the subword consisting of the values with indices in $I$. Let $\mathrm{fl}\left(\left.\pi\right|_{I}\right) \in S_{|I|}$ be the permutation with the same 
relative order as $\left.\pi\right|_{I}$. Here $f$ is the flattening operator. For example $\mathrm{f}\left(\left.31245\right|_{[2,4]}\right)=\mathrm{fl}(324)=213$. By considering the action of $d_{i}$ on $\mathrm{fl}\left(\left.\pi\right|_{[i-1, i+1]}\right)$, we may express all nontrivial actions as

$$
x 1 y \quad x 3 y,
$$

where $d_{i}$ swaps the values $x$ and $y$ in $\mathrm{fl}\left(\left.\pi\right|_{[i-1, i+1]}\right)$ while fixing all values of $\pi$ not in $[i-1, i+1]$.

Elementary dual equivalences act on standard Young tableaux via their row reading words, as shown in Figure 3 . The next theorem expresses the transitivity of this action.

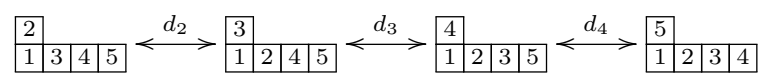

Fig. 3: The action of $d_{i}$ on $\operatorname{SYT}((4,1))$.

Theorem 2.1 (Haiman(1992, Prop. 2.4)) Two standard Young tableaux on partition shapes are dual equivalent if and only if they have the same shape.

In fact, $d_{i}$ is defined so that

$$
P \circ d_{i}(\pi)=d_{i} \circ P(\pi), \quad Q \circ d_{i}(\pi)=Q(\pi), \quad \text { and } \quad \operatorname{ID}(P(\pi))=\operatorname{ID}(\pi) .
$$

The Knuth equivalences act similarly on permutations. To perform $K_{i}$, consider $\pi_{[i-1, i+1]}$ written using the values $x<y<z$. Then $K_{i}$ permutes the values in $\pi_{[i-1, i+1]}$ by taking $y x z$ to $y z x ; x z y$ to $z x y$; and fixing $x y z$ as well as $z y x$. For example, $K_{4}(42153)=42513$. These involutions interact with the RSK correspondence by

$$
P \circ K_{i}(\pi)=P(\pi) \quad \text { and } \quad Q \circ K_{i}(\pi)=d_{i} \circ Q(\pi) .
$$

A more general treatment of dual equivalence may be found in (Sagan 2001).

Tableaux and permutations are often used in symmetric function calculations. For our purposes, it is enough to define the symmetric functions of degree $n$ as the real vector space generated by the basis of Schur functions $s_{\lambda}$, where $\lambda \vdash n$. We will additionally care about the spanning set of composition Schur functions, $s_{\alpha}$, where $\alpha \vDash n$. Each $s_{\alpha}$ is equal to $0, s_{\lambda}$, or $-s_{\lambda}$ for a unique $\lambda \vdash n$ attained from $\alpha$. To be more specific, if $\beta$ is the result of taking $\alpha$ and replacing some $\left(\alpha_{i}, \alpha_{i+1}\right)$ with $\left(\alpha_{i+1}-1, \alpha_{i}+1\right)$, then $s_{\alpha}=-s_{\beta}$. In particular, if there exists an $i$ such that $\alpha_{i}+1=\alpha_{i+1}$, then $s_{\alpha}=0$. If $s_{\alpha} \neq 0$, a series of such swaps of $\alpha_{i}$ and $\alpha_{i+1}$ may always be used to transform $\alpha$ into a unique partition $\lambda$.

The relationship between composition Schur functions and (partition) Schur functions is sometimes called the slinky correspondence for the following reason. If $\alpha$ is represented by a diagram and $s_{\alpha} \neq 0$, then we may relate $s_{\alpha}$ to a unique $s_{\lambda}$ by letting gravity pull each row down to create a partition shape, as in Figure 4 If this process does not yield a partition, the result is 0 . Otherwise, the partition shape is $\lambda$, and the sign in front of the Schur function $s_{\lambda}$ is $(-1)^{d}$, where $d$ is the sum of the number or rows each part of the composition was shifted down to make the partition. The term slinky is meant to evoke how each row slinks downward. The following property of composition Schur functions will be crucial for our purposes.

Lemma 2.2 (Egge, Loehr, and Warrington (2010)) If $f$ is a symmetric function and $c_{\alpha}$ are constants such that

$$
f=\sum_{\alpha \models n} c_{\alpha} F_{\alpha}, \quad \text { then } \quad f=\sum_{\alpha \models n} c_{\alpha} s_{\alpha}
$$




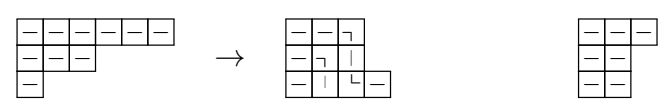

Fig. 4: The slinky correspondence showing $s_{(1,3,6)}=(-1)^{3} s_{(4,3,3)}=-s_{(4,3,3)}$ and $s_{(2,2,3)}=0$.

\subsection{The equivalence relations on tableaux}

Definition 2.3 Consider $T \in \operatorname{SYT}(\lambda)$. Define $\operatorname{sink}(T)$ and $\operatorname{slink}_{*}(T)$ as follows.

- Let $j$ be the minimal number such that the first $j$ runs of $T$ do not form a superstandard tableau. If such a $j$ does not exist $\left(T=U_{\lambda}\right)$, then slink and $\operatorname{slink}_{*}$ act as the identity.

- Construct $\operatorname{sink}(T) \in \operatorname{SYT}(\lambda)$ by permuting the cells of the $j-1$ and $j^{\text {th }}$ runs that occur below the $j^{\text {th }}$ row, giving the first $\beta_{j}(T)-1$ of these cells to the $j-1^{\text {th }}$ run. In particular,

$$
\beta_{j-1, j}(\operatorname{slink}(T))=\left(\beta_{j}(T)-1, \beta_{j-1}(T)+1\right) .
$$

- Let $\mu$ be the shape of the first $j$ runs of $T$, and let $i$ be the minimal number such that

$$
\mu_{i+1}(T) \leq \beta_{j}(T)+i-j .
$$

- Construct $\operatorname{slink}_{*}(T) \in \operatorname{SYT}(\lambda)$ by permuting the cells of the $i^{\text {th }}$ and $j^{\text {th }}$ runs that occur below the $j^{\text {th }}$ row, giving the first $\beta_{j}(T)+i-j$ of these cells the $i^{t h}$ run. In particular,

$$
\beta_{i, j}\left(\operatorname{slink}_{*}(T)\right)=\left(\beta_{j}(T)+i-j, \beta_{i}(T)+j-i\right) .
$$

Notice that the definition above refers to permuting cells in runs, dictating what the values in those cells

\begin{tabular}{|c|c|}
\hline 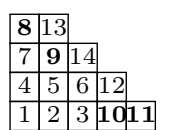 & 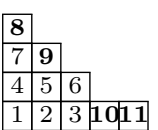 \\
\hline
\end{tabular}
will have to be. See Figures 5 and 6 for examples. A careful inspection of these definitions lead to the following.

Fig. 5: A standard young tableau followed by its restriction to the cells of $\mu$. The values in the $j^{\text {th }}$ run are bold face. Here, $i=2$ and $j=4$, so that $\mu_{i+1}=2 \leq 4+2-4=\beta_{j}+i-j$.

Lemma 2.4 For any partition $\lambda$ and $T \in \operatorname{SYT}(\lambda)$ with $i$ and $j$ as in Definition 2.3. the following hold.

1. $\operatorname{slink}(T)$ and $\operatorname{slink}_{*}(T)$ are well defined and in $\operatorname{SYT}(\lambda)$.

2. If $T \neq U_{\lambda}$, then $s_{\beta(\operatorname{sink}(T))}=-s_{\beta(T)}$ and $s_{\beta\left(\operatorname{sink}_{*}(T)\right)}=-s_{\beta(T)}$.

3. For any $T \in \operatorname{SYT}(n)$, $\operatorname{slink}_{*} \circ \operatorname{slink}_{*}(T)=T$.

4. $\operatorname{slink} \circ \operatorname{slink}_{*}(T)=\operatorname{slink}_{*} \circ \operatorname{slink}(T)$ and $\operatorname{slink}^{j-i}(T)=\operatorname{slink}^{j-i-1} \circ \operatorname{slink}_{*}(T)$.

Most the results of this abstract are consequences of Lemma 2.4. whose proof is a careful analysis of Definition 2.3 and Lemma2.2. 

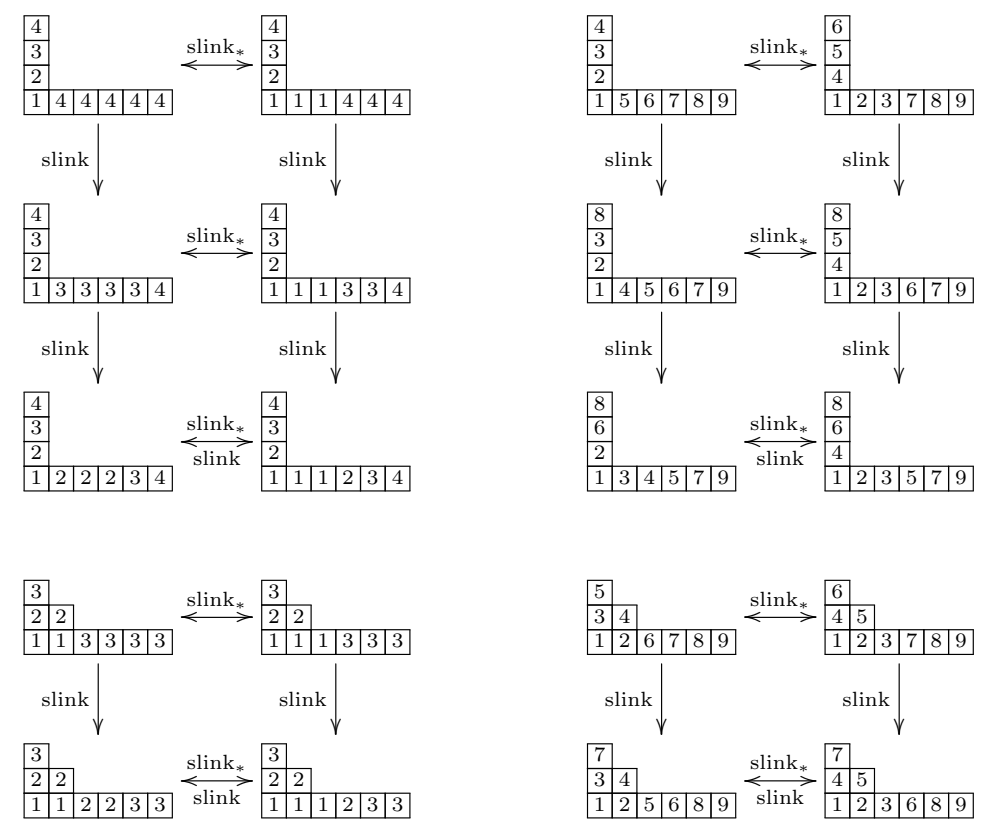

Fig. 6: Two equivalence classes of $\equiv^{1}$ using $\operatorname{SYT}(\lambda)$ on the right and their representation in UYT $(\lambda)$ on the left. The $\equiv{ }^{0}$ classes are the vertex sets of edges labeled $\operatorname{slink}_{*}$.

Definition 2.5 Define $\equiv^{0}$ and $\equiv^{1}$ as the equivalence relations on standard Young tableaux generated by $\operatorname{slink}_{*}$ and slink, respectively.

Examples of equivalence classes can be found in Figure 6 . Next, we add another equivalence relation, which we term restricted dual equivalence.

Definition 2.6 For any $T \in \operatorname{SYT}(n)$ and any $i \in[2, n-2]$, let

$$
d_{i}^{R}(T)= \begin{cases}T & i+1 \in \operatorname{ID}(T) \cap \operatorname{ID}\left(d_{i}(T)\right), \\ d_{i}(T) & \text { otherwise. }\end{cases}
$$

Further, let $\equiv^{2}$ be the equivalence relation on standard Young tableaux generated by the action of all $d_{i}^{R}$.

Theorem 2.7 The equivalence relation $\equiv^{0}$ is a refinement of $\equiv^{1}$, which in turn refines $\equiv^{2}$.

Lemma 2.8 If $\mathcal{C}$ is any union of equivalence classes of $\equiv^{k}$ with $k=0,1,2$, then

$$
\sum_{T \in \mathcal{C}} s_{\beta(T)}=\sum_{U_{\lambda} \in \mathcal{C}} s_{\lambda}
$$

Proof of Theorem 1.1: Let $k=0,1$ or 2, and let $\mathcal{C}$ be the disjoint union of equivalence classes of $\equiv^{k}$ such that $f=\sum_{T \in \mathcal{C}} F_{I D(T)}$ is symmetric. Let $c_{\lambda}$ is the multiplicity of $U_{\lambda}$ in $\mathcal{C}$. By Lemmas 2.2 and 2.8 .

$$
f=\sum_{T \in \mathcal{C}} s_{\beta(T)}=\sum_{\lambda \vdash n} c_{\lambda} s_{\lambda}
$$




\subsection{The equivalence relations on permutations}

Recall that a function defined on standard Young tableaux acts on permutations via insertion tableaux by using the RSK correspondence, acting on the insertion tableaux and fixing the recording tableaux.

Definition 2.9 Let slink, slink ${ }_{*}$, and $d_{i}^{R}$ act on permutations via insertion tableaux. For $k=0,1,2$, let $\pi \equiv{ }^{k} \pi^{\prime}$ if $P(\pi) \equiv{ }^{k} P\left(\pi^{\prime}\right)$ and $Q(\pi)=Q\left(\pi^{\prime}\right)$.

Notice that, $\equiv^{k}$ is defined so that the same function generates equivalence classes on permutations as on tableaux. In light of $(6), \equiv^{2}$ is defined on permutations by the natural action of $d_{i}^{R}$ on permutations. That is, $d_{i}^{R}(\pi)=d_{i}(\pi)$ unless $i+1 \in \operatorname{ID}(\pi) \cap \operatorname{ID}\left(d_{i}(\pi)\right)$, in which case $d_{i}^{R}$ acts trivially. By considering the action of $d_{i}^{R}$ on $\mathrm{fl}\left(\left.\pi\right|_{[i-1, i+2]}\right)$, we may express all nontrivial actions as

$$
x 1 y 4 \quad x 14 y \quad x 41 y \quad x 3 y 4 \quad x 34 y
$$

where $d_{i}^{R}$ swaps the values $x$ and $y$ in $\mathrm{fl}\left(\left.\pi\right|_{[i-1, i+2]}\right)$ while fixing values of $\pi$ not in $[i-1, i+1]$.

Proposition 2.10 The action of $K_{j}$ on permutations commutes with $d_{i}^{R}$, slink, and $\operatorname{slink}_{*}$.

Corollary 2.11 For any $k=0,1,2$, let $\mathcal{C} \subset S_{n}$ be a union of equivalence classes of $\equiv^{k}$ such that $f=\sum_{\pi \in \mathcal{C}} F_{\mathrm{ID}(\pi)}$ is a symmetric function. Then

$$
f=\sum_{\lambda \vdash n}|\{\pi \in \mathcal{C}: \pi \in \operatorname{SYam}(\lambda)\}| \cdot s_{\lambda} .
$$

We end this section by considering generating functions of $\equiv^{k}$ classes.

Definition 2.12 For $k=0,1,2$, let $\left\{f^{(k)}\right\}$ be the set of functions that can be realized as $\sum_{T \in \mathcal{C}} F_{\operatorname{ID}(T)}$, where $\mathcal{C}$ is a single equivalence class of $\equiv^{k}$.

We use the notation $\left\{f^{(k)}\right\}$ because it is currently unknown how to best index these sets. In the next section, we will show that each $\left\{f^{(k)}\right\}$ forms a spanning set of the quasisymmetric functions.

Conjecture 2.13 The set $\left\{f^{(2)}\right\}$ forms a basis for the quasisymmetric functions.

This conjecture has been verified up to degree 11 with the aid of a computer.

Example 2.14 Consider the collection of $\equiv^{0}$ classes and its associated quasisymmetric function below.

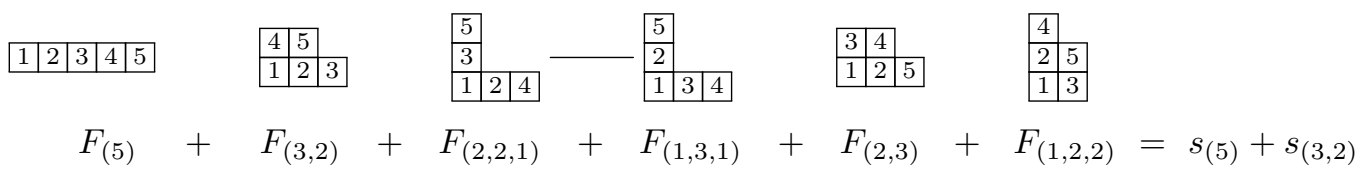

The sum can be shown to be symmetric, and so we may apply Theorem 1.1 to find the Schur expansion directly. Alternatively, if we wish to use $\equiv^{2}$ classes, then Conjecture 2.13 implies that there is only one way to achieve the same function, as shown below.

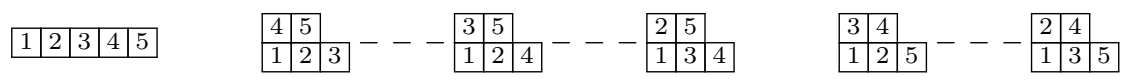




\section{Extending to the quasisymmetric Schur functions}

A standard reverse composition tableau $T$ is a standard filling of a composition shape that is increasing down the first column, decreasing across rows from left to right, and that satisfies the following additional property. Given any two values $a>b$ that are adjacent in a row of $T$, no value in $(b, a)$ may occur below and in the same column as $b$. If $a$ is the furthest right value in its row, treat the entry immediately to its right as 0 . The set of standard reverse composition tableaux is denoted $\operatorname{SRCT}(\alpha)$. The bent reading word of $T \in \operatorname{SRCT}(\alpha)$, bw $(T)$, is given by reading down columns from right to left and then up the leftmost column. Examples are given in Figure 8. We may now introduce the quasisymmetric Schur functions, originally appearing in (Haglund et al., 2011), by

$$
\mathcal{S}_{\alpha}=\sum_{T \in \operatorname{SRCT}(\alpha)} F_{\mathrm{ID}(\mathrm{bw}(T))}
$$

Importantly, $\left\{\mathcal{S}_{\alpha}\right\}$ is a basis for the quasisymmetric functions.

Remark 3.1 Traditionally, the inverse descent set of $T \in \operatorname{SRCT}(\alpha)$ is the set of $i$ such that $i+1$ is in a column weakly to the right of $i$. This is equivalent to our defition.

A standard reverse Young tableau of partition shape $\lambda$ is a standard filling of shape $\lambda$ that is decreasing up columns and across rows from left to right. The set of all standard reverse Young tableau is denoted $\operatorname{SRT}(\lambda)$. The reverse column word of $T \in \operatorname{SRT}(\lambda), \operatorname{cw}(T)$, will be the reverse of the normal convention, proceeding up columns, right to left. It can then be shown (see (Luoto et al., 2013, Ch. 3.2)) that the basis of Schur functions may be rewritten as

$$
s_{\lambda}=\sum_{T \in \operatorname{SRT}(\lambda)} F_{\mathrm{ID}(\mathrm{cw}(T))} .
$$

The set $\mathcal{C}(\alpha) \subset \operatorname{SRT}(\lambda(\alpha))$ is given by applying Mason's bijection to $\operatorname{SRCT}(\alpha)$, sorting the columns, and bottom justifying. We denote this map by $\rho$. Importantly, $\rho$ preserves the set of values in each column. Further, it preserves inverse descent sets, since both SRT and SRCT have the property that $i$ is an inverse descent of a filling if and only if $i+1$ is in a column weakly to its right. Thus,

$$
\mathcal{S}_{\alpha}=\sum_{T \in \mathcal{C}(\alpha)} F_{\mathrm{ID}(T)}
$$

The original definition of $\rho$, along with its inverse, is in (Mason 2008). Figure 8 gives examples.

For any cell $c$ of $\alpha$, the set of cells weakly below $c$ in its column or weakly above $c$ in the column to its left is called an $\alpha$-pistol, as in Figure 7
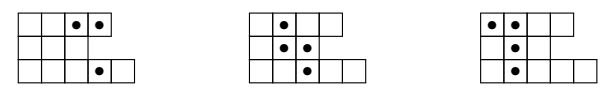

Fig. 7: Three $\alpha$-pistols of $\alpha=(5,3,4,3)$.

Let $\tilde{d}_{i}: S_{n} \rightarrow S_{n}$ be the involution that cyclically permutes the values $i-1, i$, and $i+1$ as

$$
\begin{aligned}
& \tilde{d}_{i}(\ldots i \ldots i-1 \ldots i+1 \ldots)=(\ldots i-1 \ldots i+1 \ldots i \ldots), \\
& \tilde{d}_{i}(\ldots i \ldots i+1 \ldots i-1 \ldots)=(\ldots i+1 \ldots i-1 \ldots i \ldots),
\end{aligned}
$$


and acts as the identity if $i$ is positioned between $i-1$ and $i+1$. Thus, $\tilde{d}_{3} \circ \tilde{d}_{2}(4123)=\tilde{d}_{3}(4123)=3142$. Let $d_{i}$ and $\tilde{d}_{i}$ act on $\operatorname{SRCT}(\alpha)$ via the bent reading word.

We use $d_{i}$ and $\tilde{d}_{i}$ to define a transitive family of involutions on $\operatorname{SRCT}(\alpha)$.

$$
D_{i}^{Q}(T)= \begin{cases}T & \text { if } i-1, i, \text { and } i+1 \text { are contained in two cells of the first column } \\ \tilde{d}_{i}(T) & \text { and one in the second column, } i-1, i, \text { and } i+1 \text { are contained in an } \alpha \text {-pistol, } \\ d_{i}(T) & \text { otherwise. }\end{cases}
$$

As an example, we may take $\mathrm{bw}(T)=\pi=3265874$ and $\alpha=(3,2,3)$ as in Figure 8. Examples of each of the three cases are as follows. First, $D_{7}^{Q}(T)=T$. Then, $D_{6}^{Q}(T)=\tilde{d}_{6}(T)$, which has bent reading word 3257468. Finally, $D_{3}^{Q}(T)=d_{3}(T)$, which has bent reading word 4265873.

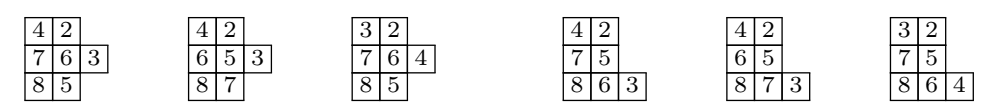

Fig. 8: Three standard reverse composition tableaux of shape $\alpha=(2,3,2)$ followed by their image via Mason's bijection. From the left, $T \in \operatorname{SRCT}(\alpha)$ with bent reading word $\pi=3265874$, followed by $D_{6}^{Q}(T)$, and then $D_{3}^{Q}(T)$.

Proposition 3.2 The set of involutions $D_{i}^{Q}$ preserves and acts transitively on $\operatorname{SRCT}(\alpha)$.

We want to express the action of $D_{i}^{Q}$ on $\mathcal{C}(\alpha)$ via Mason's bijection. Given $T \in \operatorname{SRT}(\lambda)$, define the elementary quasi-dual equivalence relations as

$$
d_{i}^{Q}(T)= \begin{cases}T & \text { if } i-1, i, \text { and } i+1 \text { are in two cells of the first column and one } \\ d_{i}(T) & \text { in the second column }\end{cases}
$$

As with earlier involutions, we define the action of $d_{i}^{Q}$ on a permutation via insertion tableaux.

Proposition 3.3 For any $T \in \operatorname{SRCT}(\alpha)$,

$$
d_{i}^{Q} \circ \rho(T)=\rho \circ D_{i}^{Q}(T)
$$

In particular, the set of $d_{i}^{Q}$ preserve and act transitively on $\mathcal{C}(\alpha)$.

Remark 3.4 In (Bessenrodt et al., 2011), there is a similar analysis of the relationship between $\rho$ and dual equivalence on SRT to prove a quasisymmetric Littlewood-Richardson rule. We have made these relationships more explicit by introducing $d_{i}^{Q}$ and $D_{i}^{Q}$.

Recall from Definition 2.12 that $\left\{f^{(k)}\right\}$ is the set of functions of the form $f=\sum_{T \in C} F_{\mathrm{ID}(T)}$, where $\mathcal{C}$ is any $\equiv^{k}$ class. Each $\equiv^{k}$ also defines relations on $\operatorname{SRT}(\lambda)$ via the reverse column reading word. In this way, it makes sense to compare the equivalence classes generated by $d_{i}^{Q}$ to the classes of $\equiv^{k}$ and to compare $\left\{\mathcal{S}_{\alpha}\right\}$ to $\left\{f^{(k)}\right\}$.

Theorem 3.5 Quasi-dual equivalence is a coarsening of $\equiv^{k}$, for each $k=0,1,2$. In particular, each quasisymmetric Schur function is a nonnegative sum of functions in $\left\{f^{(k)}\right\}$, for each $k=0,1,2$. In particular, any symmetric function that is a positive sum of quasisymmetric Schur functions is Schur positive. 
The last statement of Theorem 3.5 was previously stated in (Bessenrodt et al. 2014. Lemma 2.21) as a corollary to results in (Haglund et al. 2011). Though we have provided a new proof, it is this past result that motivated much of the research in this abstract.

Corollary 3.6 For each $k=0,1,2$, the set of functions $\left\{f^{(k)}\right\}$ of the form $f=\sum_{T \in C} F_{\mathrm{ID}(T)}$ forms a spanning set of the quasisymmetric functions.

\section{Extending to shifted dual equivalence}

A partition $\lambda$ is termed a strict partition if $\lambda=\left(\lambda_{1}>\lambda_{2}>\ldots>\lambda_{k}>0\right)$, the shifted shape $\lambda$ is the set of squares in positions $\left\{(i, j)\right.$ given $\left.1 \leq i \leq k, i \leq j \leq \lambda_{i}+i-1\right\}$. A standard shifted tableau $T$ is a standard filling of a shifted shape that is increasing up columns and across rows from left to right. For example, see Figure 9 Let $\operatorname{SST}(\lambda)$ be the set of standard shifted tableaux of shifted shape $\lambda$.

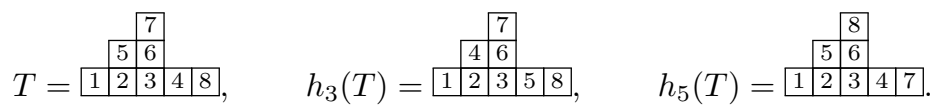

Fig. 9: Three shifted dual equivalent standard shifted tableau of shape $\lambda=(5,2,1)$.

Definition 4.1 ((Haiman, 1992) $)$ Given a permutation $\pi \in S_{n}$, define the elementary shifted dual equivalence $h_{i}$ for all $1 \leq i \leq n-3$ as follows. If $n \leq 3$, then $h_{1}(\pi)=\pi$. If $n=4$, then $h_{1}(\pi)$ acts by swapping $x$ and $y$ in the cases below,

$$
1 x 2 y \quad x 12 y \quad 1 x 4 y \quad x 14 y \quad 4 x 1 y \quad x 41 y \quad 4 x 3 y \quad x 43 y
$$

and $h_{1}(\pi)=\pi$ otherwise. If $n>4$, then $h_{i}$ is the involution that fixes values not in $I=[i, i+3]$ and permutes the values in I via $\mathrm{fl}\left(\left.h_{i}(\pi)\right|_{I}\right)=h_{1}\left(\mathrm{fl}\left(\left.\pi\right|_{I}\right)\right)$.

As an example, $h_{1}(24531)=14532, h_{2}(25134)=24135$, and $h_{3}(314526)=314526$. Notice that every nontrivial action of $h_{i}$ is equal to either $d_{i+1}$ or $d_{i+2}$. Two permutations are shifted dual equivalent if they are connected by some sequence of elementary shifted dual equivalences. Given a standard shifted tableau $T$, we define $h_{i}(T)$ as the result of letting $h_{i}$ act on the row reading word of $T$. We can define an equivalence relation on standard shifted tableaux by saying $T$ and $h_{i}(T)$ are shifted dual equivalent for all $i$. See Figure 9 for an example.

Theorem 4.2 (Prop. 2.4, (Haiman, 1992)) For all strict partitions $\lambda$, the set of $h_{i}$ act transitively on $\operatorname{SSYT}(\lambda)$.

Definition 4.3 Let $\left\{f^{(h)}\right\}$ be the set of functions that can be realized as $\sum_{T \in \mathcal{C}} F_{\mathrm{ID}(T)}$, where $\mathcal{C}$ is a single shifted dual equivalence class of permutations.

To study $f^{(h)}$, we need some intermediary involutions. For an inverse descent set $D \subset[n-1]$, let $D^{C}$ be the compliment of $D$ in $[n-1]$. The involution on quasisymmetric functions that sends $F_{D}$ to $F_{D^{C}}$ and $s_{\lambda}$ to $s_{\lambda^{\prime}}$ is denoted $\omega$. Given $\pi \in S_{n}$, let the reverse of $\pi, \operatorname{rev}(\pi)$, be the result of reading $\pi$ from right to left. Let the flip of $\pi$, flip $(\pi)$, be the result of taking $\operatorname{rev}(\pi)$ and inverting all values, sending $i$ to $n+1-i$. For example, $\operatorname{rev}(21435)=53412$ and $\operatorname{flip}(21435)=13254$. Notice that $\operatorname{ID}(\operatorname{rev}(\pi))=\operatorname{ID}(\pi)^{C}$, and $\operatorname{ID}(\operatorname{flip}(\pi))$ is the result of sending each $i \in \operatorname{ID}(\pi)$ to $n-i$. 
Proposition 4.4 Given distinct $\pi, \pi^{\prime} \in S_{n}$, if $d_{i}^{R}(\operatorname{rev}(\pi))=\operatorname{rev}\left(\pi^{\prime}\right)$, then $h_{i-1}(\pi)=\pi^{\prime}$. If $d_{i}^{R}(\operatorname{flip}(\pi))=$ flip $\left(\pi^{\prime}\right)$, then $h_{n-i}(\pi)=\pi^{\prime}$.

Theorem 4.5 For $k=0,1,2$, the relation $\equiv^{k}$ on $S_{n}$ is a refinement of shifted dual equivalence on the reverse of the permutations in $S_{n}$. In particular, every function in $\left\{f^{(h)}\right\}$ is a nonnegative sum of functions in $\left\{\omega(f): f \in\left\{f^{k}\right\}\right\}$, for each $k=0,1,2$. Further, the relation $\equiv^{k}$ on $S_{n}$ is a refinement of the relation generated by shifted dual equivalence on the flip of the permutations in $S_{n}$.

Corollary 4.6 Let $\mathcal{C}$ be any union of shifted dual equivalence classes of $S_{n}$ such that $f=\sum_{\pi \in \mathcal{C}} F_{\mathrm{ID}(\pi)}$ is symmetric. Then

$$
f=\sum_{\lambda \vdash n}|\{\pi \in \mathcal{C}: \operatorname{rev}(\pi) \in \operatorname{SYam}(\lambda)\}| \cdot s_{\lambda^{\prime}}=\sum_{\lambda \vdash n}|\{\pi \in \mathcal{C}: \operatorname{flip}(\pi) \in \operatorname{SYam}(\lambda)\}| \cdot s_{\lambda} .
$$

Remark 4.7 Traditionally, the generating function over standard shifted tableaux uses peak functions. It is thus surprising to find a simple condition for Schur positivity with the generating functions given above.

Our final result gives a converse to Proposition 4.4 , so long as we only consider the row reading words of shifted tableaux in $\operatorname{SSYT}(\lambda)$.

Proposition 4.8 For all strict partitions $\lambda$, the set of $d_{i}^{R}$ acts transitively on $\operatorname{SSYT}(\lambda)$ via the flip of the row reading word.

\section{References}

C. Bessenrodt, K. Luoto, and S. van Willigenburg. Skew quasisymmetric Schur functions and noncommutative Schur functions. Advances in Mathematics, 226(5):4492-4532, 2011.

C. Bessenrodt, V. V. Tewari, and S. J. van Willigenburg. Symmetric skew quasisymmetric Schur functions. arXiv preprint arXiv:1410.2934, 2014.

E. Egge, N. A. Loehr, and G. S. Warrington. From quasisymmetric expansions to Schur expansions via a modified inverse Kostka matrix. European Journal of Combinatorics, 31(8):2014-2027, 2010.

I. M. Gessel. Multipartite P-partitions and inner products of skew Schur functions. Contemp. Math, 34: 289-317, 1984.

J. Haglund, K. Luoto, S. Mason, and S. van Willigenburg. Quasisymmetric Schur functions. Journal of Combinatorial Theory, Series A, 118(2):463-490, 2011.

M. D. Haiman. Dual equivalence with applications, including a conjecture of Proctor. Discrete Math, 99 (1):79-113, 1992.

K. Luoto, S. Mykytiuk, and S. van Willigenburg. An introduction to quasisymmetric Schur functions. Hopf algebras, quasisymmetric functions, and Young composition tableaux. New York, NY: Springer, 2013. doi: 10.1007/978-1-4614-7300-8.

S. Mason. A decomposition of Schur functions and an analogue of the Robinson-Schensted-Knuth algorithm. Séminaire Lotharingien de Combinatoire, 57:B57e, 2008.

B. E. Sagan. The Symmetric Group: Representations, Combinatorial Algorithms, and Symmetric Functions, volume 203 of Graduate Texts in Mathematics. Springer-Verlag, New York, second edition, 2001. 